\title{
238.
}

\section{NOTE SUR LES NORMALES D'UNE CONIQUE.}

[From the Journal für die reine und angewandte Mathematik, (Crelle), tom. LVI. (1859), pp. 182-185.]

ON connaît les recherches très élégantes de M. Joachimsthal sur les normales d'une conique [voir le Mémoire "Ueber die Normalen der Ellipse und des Ellipsoids," Crelle, t. XXVI. (1843) pp. 172-180]; en particulier l'auteur a obtenu le théorème suivant: en supposant que 1, 2, 3, 4 soient des points d'une eonique tels que les quatre normales se rencontrent dans un même point, on prend le pôle de la droite $(1,2)$ par rapport à la conique et on mène par ce pôle des perpendiculaires aux diamètres de la conique; celà étant, en prenant sur chaque diamètre dans le sens opposé un point dont la distance du centre est égale à la distance du pied de la perpendiculaire sur ce même diamètre, la droite menée par ces deux points passe par les deux points 3 et 4 . Mais cette propriété peut s'énoncer d'une manière beaucoup plus simple; la droite dont il s'agit est la polaire (ou autrement dit l'harmonicale)-par rapport au triangle formé par les deux diamètres et la droite située à l'infini-du pôle de la droite $(1,2)$ par rapport à la conique. Or on sait que l'idée de la perpendiculaire peut être généralisée. Savoir en prenant une conique quelconque que nous appelons la conique absolue, deux droites harmoniques par rapport à cette conique peuvent être appelées perpendiculaires (et de même deux points harmoniques par rapport à la conique absolue peuvent être appelés perpendiculaires). Cela posé, on peut parler dans un sens plus général des normales, etc. d'une courbe quelconque. En effet, que l'on s'imagine comme auparavant (outre la conique absolue) une conique donnée quelconque et quatre points 1, 2, 3 et 4 de cette conique tels que les normales se rencontrent dans un même point. Au lieu du triangle ci-dessus mentionné on a le triangle formé par les trois axes harmoniques (ou autrement dit, conjugués) communs aux deux coniques, et le théorème peut s'énoncer comme suit: En prenant le pôle de la droite $(1,2)$ par rapport à la conique donnée et puis la polaire (l'harmonicale) de ce pôle par rapport aux axes conjugués de la conique donnée et de la conique absolue, cette polaire passe par les deux points 
3 et 4. Ou (ce qui revient à la même chose) on peut considérer les points $1,2,3,4$ comme les angles d'un quadrilatère inscrit dans la conique donnée, les quatre tangentes à cette conique aux points dont il s'agit seront les côtés d'un quadrilatère circonscrit à la conique donnée; cela étant, les six côtés du quadrilatère inscrit seront les polaires (les harmonicales) - par rapport aux trois axes conjugués de la conique donnée et de la conique absolue-des six sommets du quadrilatère circonscrit.

Pour démontrer cela, je fais observer qu'il est permis de rapporter la conique absolue et la conique donnée aux trois axes conjugués communs, c'est-à-dire de prendre

$$
x^{2}+y^{2}+z^{2}=0,
$$

pour équation de la conique absolue, et

$$
a x^{2}+b y^{2}+c z^{2}=0,
$$

pour équation de la conique donnée: cela posé (et en observant que, d'après la définition, deux droites $A x+B y+C z=0, A^{\prime} x+B^{\prime} y+C^{\prime} z=0$ seront perpendiculaires si $\left.A A^{\prime}+B B^{\prime}+C C^{\prime}=0\right)$ on obtient sans peine

$$
\frac{x(b-c)}{x_{1}}+\frac{y(c-a)}{y_{1}}+\frac{z(a-b)}{z_{1}}=0,
$$

pour équation de la normale au point 1 , en désignant par $\left(x_{1}, y_{1}, z_{1}\right)$ les coordonnées de ce point. On a de même, en désignant par $\left(x_{2}, y_{2}, z_{2}\right)$ et $\left(x_{3}, y_{3}, z_{3}\right)$ les coordonnées des points 2 et 3 ,

$$
\begin{aligned}
& \frac{x(b-c)}{x_{2}}+\frac{y(c-a)}{y_{2}}+\frac{z(a-b)}{z_{2}}=0, \\
& \frac{x(b-c)}{x_{3}}+\frac{y(c-a)}{y_{3}}+\frac{z(a-b)}{z_{3}}=0,
\end{aligned}
$$

pour les équations des normales aux points 2 et 3 ; et la condition qui exprime que ces trois normales se rencontrent dans un même point sera évidemment

$$
\left|\begin{array}{ccc}
\frac{1}{x_{1}}, & \frac{1}{y_{1}}, & \frac{1}{z_{1}} \\
\frac{1}{x_{2}}, & \frac{1}{y_{2}}, & \frac{1}{z_{2}} \\
\frac{1}{x_{3}}, & \frac{1}{y_{3}}, & \frac{1}{z_{3}}
\end{array}\right|=0 .
$$

Mais les coordonnées $\left(x_{1}, y_{1}, z_{1}\right)$ etc. satisfont à l'équation $a x^{2}+b y^{2}+c z^{2}=0$, on a donc aussi

$$
\left|\begin{array}{lll}
x_{1}{ }^{2}, & y_{1}{ }^{2}, & z_{1}{ }^{2} \\
x_{2}{ }^{2}, & y_{2}{ }^{2}, & z_{2}{ }^{2} \\
x_{3}{ }^{2}, & y_{3}{ }^{2}, & z_{3}{ }^{2}
\end{array}\right|=0
$$


et de ces deux équations on déduit la suivante

$$
\left\{\begin{array}{lll}
x_{1}, & y_{1}, & z_{1} \\
x_{2}, & y_{2}, & z_{2} \\
x_{3}, & y_{3}, & z_{3}
\end{array}\right\}=0
$$

en désignant par le symbole qui forme le premier membre la fonction

$$
x_{1} y_{2} z_{3}+x_{1} y_{3} z_{2}+x_{2} y_{3} z_{1}+x_{2} y_{1} z_{3}+x_{3} y_{1} z_{2}+x_{3} y_{2} z_{1}
$$

C'est ce qui résulte de l'identité

$$
\left\{\begin{array}{lll}
x_{1}, & y_{1}, & z_{1} \\
x_{2}, & y_{2}, & z_{2} \\
x_{3}, & y_{3}, & z_{3}
\end{array}\right\} \times\left|\begin{array}{ccc}
x_{1}, & y_{1}, & z_{1} \\
x_{2}, & y_{2}, & z_{2} \\
x_{3}, & y_{3}, & z_{3}
\end{array}\right|=\left|\begin{array}{ccc}
x_{1}{ }^{2}, & y_{1}{ }^{2}, & z_{1}{ }^{2} \\
x_{2}{ }^{2}, & y_{2}{ }^{2}, & z_{2}{ }^{2} \\
x_{3}{ }^{2}, & y_{3}{ }^{2}, & z_{3}{ }^{2}
\end{array}\right|+2 x_{1} y_{1} z_{1} x_{2} y_{2} z_{2} x_{3} y_{3} z_{3}\left|\begin{array}{ccc}
\frac{1}{x_{1}}, & \frac{1}{y_{1}}, & \frac{1}{z_{1}} \\
\frac{1}{x_{2}}, & \frac{1}{y_{2}}, & \frac{1}{z_{2}} \\
\frac{1}{x_{3}}, & \frac{1}{y_{3}}, & \frac{1}{z_{3}}
\end{array}\right| ;
$$

car le déterminant qui forme le second facteur du premier membre de l'équation ne s'évanouissant pas, c'est l'autre facteur qui devra s'évanouir en vertu des deux relations données.

L'équation de la droite $(1,2)$ sera

$$
\left|\begin{array}{lll}
x, & y, & z \\
x_{1}, & y_{1}, & z_{1} \\
x_{2}, & y_{2}, & z_{2}
\end{array}\right|=0:
$$

les coordonnées du pôle de cette droite par rapport à la conique donnée $a x^{2}+b y^{2}+c z^{2}=0$, seront

$$
\frac{1}{a}\left(y_{1} z_{2}-y_{2} z_{1}\right): \frac{1}{b}\left(z_{1} x_{2}-z_{2} x_{1}\right): \frac{1}{c}\left(x_{1} y_{2}-x_{2} y_{1}\right)
$$

mais les deux équations $a x_{1}{ }^{2}+b y_{1}{ }^{2}+c z_{1}{ }^{2}=0, a x_{2}{ }^{2}+b y_{2}{ }^{2}+c z_{2}{ }^{2}=0$ donnent $a: b: c$ $=y_{1}{ }^{2} z_{2}{ }^{2}-y_{2}{ }^{2} z_{1}{ }^{2}: z_{1}{ }^{2} x_{2}{ }^{2}-z_{2}{ }^{2} x_{1}{ }^{2}: x_{1}{ }^{2} y_{2}{ }^{2}-x_{2}{ }^{2} y_{1}{ }^{2} ;$ par suite de cela les coordonneés du pôle deviennent

$$
\frac{1}{y_{1} z_{2}+y_{2} z_{1}}: \frac{1}{z_{1} x_{2}+z_{2} x_{1}}: \frac{1}{x_{1} y_{2}+x_{2} y_{1}}
$$

donc l'équation de la polaire (l'harmonicale) de ce pôle par rapport aux trois droites $(x=0, y=0, z=0)$ sera

$$
x\left(y_{1} z_{2}+y_{2} z_{1}\right)+y\left(z_{1} x_{2}+z_{2} x_{1}\right)+z\left(x_{1} y_{2}+x_{2} y_{1}\right)=0,
$$

laquelle peut être représentée comme suit

$$
\left\{\begin{array}{lll}
x, & y, & z \\
x_{1}, & y_{1}, & z_{1} \\
x_{2}, & y_{2}, & z_{2}
\end{array}\right\}=0,
$$


et, en substituant $\left(x_{3}, y_{3}, z_{3}\right)$ au lieu de $(x, y, z)$, on voit que la droite dont il s'agit contient le point 3. De même cette droite contient le point 4, de sorte que le théorème se trouve démontré.

Observons encore que dans la géométrie de la sphère on peut prendre pour la conique absolue la conique imaginaire qui est l'intersection de la surface sphérique avec la surface conique imaginaire $x^{2}+y^{2}+z^{2}=0$. Le mot perpendiculaire aura alors la signification ordinaire, et on aura pour les coniques sphériques ce théorème très simple, savoir que les six côtés du quadrilatère inscrit seront les polaires (les harmonicales) par rapport aux trois axes de la conique sphérique donnée-des six sommets du quadrilatère circonscrit. C'est ce que l'on reconnaît aussi par l'analyse que je viens de donner, laquelle en effet est précisément celle dont on se servirait natureliement pour les coniques sphériques.

Londres, le 10 Mars, 1857. 